Research Article

\title{
Fault Diagnosis Method of the Construction Machinery Hydraulic System Based on Artificial Intelligence Dynamic Monitoring
}

\author{
Xin Zhou $(\mathbb{D}$ and Xianqing Lei \\ School of Mechatronics Engineering, Henan University of Science and Technology, Luoyang 471003, Henan, China \\ Correspondence should be addressed to Xin Zhou; 205400000003@stu.haust.edu.cn
}

Received 20 May 2021; Revised 17 June 2021; Accepted 9 July 2021; Published 16 July 2021

Academic Editor: Sang-Bing Tsai

Copyright (c) 2021 Xin Zhou and Xianqing Lei. This is an open access article distributed under the Creative Commons Attribution License, which permits unrestricted use, distribution, and reproduction in any medium, provided the original work is properly cited.

\begin{abstract}
This paper aims to study the fault diagnosis method of the mechanical hydraulic system based on artificial intelligence dynamic monitoring. According to the characteristics of functional principal component analysis (FPCA) and neural network in the fault diagnosis method in the feature extraction process, the fault diagnosis method combining functional principal component analysis and BP neural network is studied and it is applied to the fault of the coordinator hydraulic system diagnosis. This article mainly completed the following tasks: analyzing the structure and working principle of the mechanical hydraulic system, studying the failure mechanism and failure mode of the mechanical hydraulic system, summarizing the common failures of the hydraulic system and the individual failures of the mechanical hydraulic system, and establishing the mechanical hydraulic system. Description of failure mode and effects analysis (FMEA): then, a joint simulation model of the mechanical hydraulic system was established in ADAMS and AMESim, and the fault detection signal of the hydraulic system was determined and compared with the experimental data. At the same time, the simulation data of the cosimulation model were compared with the simulation data of the hydraulic model in MATLAB to further verify the correctness of the model. The functional principal component analysis is used to perform functional processing on sample data, feature parameters are extracted, and the BP neural network is used to train the mapping relationship between feature parameters and fault parameters. The consistency is verified, and the fault diagnosis method is finally completed. The experimental results show that the diagnostic accuracy rates are 0.9848 and 0.9927 , respectively, the reliability is significantly improved, close to $100 \%$, and the uncertainty is basically 0 , which significantly improves the accuracy of fault diagnosis.
\end{abstract}

\section{Introduction}

Robot technology has the following characteristics: first, it has a high degree of freedom and a strong ability to control the system. In the modern professional industry, in order to achieve high control of the system, the robot will show a high degree of freedom to meet the needs of different programs. The second is that the robot depends on the Internet of Things and big data in the process of operation technical support, through the use of Internet technology to achieve automatic control of the robot. Third, it has high computing and information feedback. At present, the research of artificial intelligence is carried out in combination with specific fields. Basically, there are the following areas: (1) expert system is a knowledge system built on the existing knowledge of human experts. It applies artificial intelligence technology and simulates the thinking process of human experts when solving problems to solve various problems in the field. The system can work as a human expert. (2) Pattern recognition is to study how to make the machine have the ability to perceive. It mainly studies the recognition of visual mode and auditory mode. In recent years, the methods of applying the fuzzy mathematical model and artificial neural network model have been rapidly developed to gradually replace the traditional identification methods using statistical and structural patterns. In particular, neural network methods have made great progress in pattern recognition. (3) Artificial neural network is inspired by studying the mystery of the human brain, trying to use a large number of processing units (artificial neurons, processing elements, 
electronic components, etc.) to imitate the human brain nervous system engineering structure and working mechanism. In artificial neural networks, the processing of information is achieved by the interaction between neurons. The storage of knowledge and information manifests as a distributed physical connection between the interconnection of network elements. The learning and recognition of the network depend on the neurons' dynamic evolution of connection weights. Artificial neural networks have become an extremely important research area in artificial intelligence.

Due to the importance of fault diagnosis of mechanical systems, many research teams have begun to study fault diagnosis of mechanical systems and have achieved good results. The electrohydraulic servo drive system is a complex system of mechanical, electrical, and hydraulic systems. If it cannot be repaired for a long time, the possibility of multiple failures must be considered. Considering this possibility, Wang proposed a multifault diagnosis method based on an extended Kalman filter (EKF). By analyzing the failure modes and mechanisms of the electrohydraulic servo drive system and modeling the selected typical failure modes, the relationship between the key parameters of the system and the failure is obtained. Extended Kalman filter is a commonly used parameter estimation algorithm for online fault diagnosis. Then, the extended Kalman filter is used to diagnose potential faults. Simulation results show that the multifault diagnosis method based on the extended Kalman filter is effective for multifault diagnosis of electrohydraulic servo drive systems $[1,2]$. The naval gun weapon system is a complex large-scale mechatronic system composed of the mechanical system, electrical system, hydraulic system, and so on. The system has a wide working range, poor working environment, and high failure rate. Whether the naval gun weapon system can work normally directly affects the performance index of the weapon system and even affects the entire naval battle situation. Chen introduced a fault diagnosis technology that organically combines neural networks and expert systems. The failure mode is analyzed theoretically and experimentally. The results show that the neural network fault diagnosis technology can be extended to the naval gun system fault diagnosis integrated system $[3,4]$. Although the research results are richer now, there are still some shortcomings, mainly due to insufficient accuracy.

In the research of artificial intelligence, an artificial neural network is a good method that can solve many problems in fault diagnosis. Therefore, it is widely used in system research. Massimo proposed an artificial neural network- (ANN-) based photovoltaic new method for system fault diagnosis. For a given set of operating conditions (solar irradiance and temperature of photovoltaic (PV) components), simulation models were used to calculate attributes such as the number of peaks in the current, voltage, and current-voltage (I-V) characteristics of the photovoltaic string. The simulated attributes are then compared with those measured on-site to identify possible faulty operating conditions. Two different algorithms were then developed to isolate and identify eight different types of faults. The method has been validated using an experimental database of climate and electrical parameters of photovoltaic cells installed at the Renewable Energy Laboratory (REL) of Jijel University (Algeria). The results show that this method can accurately detect and classify different faults in photovoltaic arrays. Massimo also introduced a method to implement this as a field programmable gate array (FPGA) using the Xilinx System Generator (XSG) and Integrated Software Environment (ISE) [5, 6]. Due to the effectiveness of artificial intelligence methods can artificial neural network methods be used to study the fault diagnosis methods of mechanical hydraulic systems and solve the problem of insufficient accuracy of current fault diagnosis.

This article first explains the current status of fault diagnosis research of hydraulic systems at home and abroad and introduces the fault diagnosis research and application status of mechanical hydraulic systems. It briefly describes the development trend of hydraulic system fault diagnosis and then details the mechanical hydraulic system structure and working principle, analyzes the failure mechanism and failure mode of the hydraulic system, summarizes the common failures of the hydraulic system and the individual failures of the mechanical hydraulic system, and establishes the FMEA form of the mechanical hydraulic system. Then, the mechanical dynamics model and the hydraulic system model are established in ADAMS and AMESim, respectively, and joint simulation is performed. According to the characteristics of mechanical hydraulic pressure, the fault detection signal is determined and compared with the experimental data to meet the requirements of fault simulation. At the same time, the joint simulation data is compared with the simulation data of the hydraulic model in MATLAB to further verify the correctness of the model. Finally, the obtained sample data is functionalized and combined with functional principal component analysis to extract the characteristic parameters of the processed data. The characteristic parameters and fault parameters are used as the input and output of the BP neural network, respectively. After data training, the feasibility is verified with some sample data. Finally, the research on fault diagnosis methods is completed.

\section{Proposed Method}

2.1. Artificial Intelligence Dynamic Monitoring. Dynamic monitoring is a complex system engineering, which adapts to the multidimensional structure of environmental science, can meet the research of environmental problems of different spatial scales and different time scales, and can serve various levels of resource investigation, development, environmental protection departments, and planning and management departments. The testing methods in the engineering industry in our country have also reached the international advanced level. In addition, with the substantial improvement in the level of construction mechanization, especially the operation of advanced mechanized means, higher requirements are put forward for roadbed detection methods, and the traditional static load detection methods can no longer be adopted. In order to improve construction efficiency and ensure construction quality and 
economic benefits, it is necessary to adopt and promote the detection method of dynamic deformation modulus.

Artificial intelligence currently has two definitions. The first refers to artificial intelligence that refers to "machine intelligence, which is any method that allows computers to pass the Turing test," and the other is defined in computer science: "a machine that mimics the "cognitive" function of the connection of human beings with other human minds." These two definitions precisely reflect the two current lines of artificial intelligence exploration. The first definition of artificial intelligence focuses on problem-solving. The development of artificial intelligence: it is not necessary to imitate the way of thinking of humans but to solve the problem according to a certain machine language; the second definition reflects the basic ideas and basic contents of the subject of artificial intelligence $[7,8]$. That is to say, artificial intelligence is the study of the laws of human intelligent activity, the construction of artificial systems with certain intelligence, and the study of how to allow computers to complete tasks that previously required human intelligence to be competent, that is, the study of how to use computer software and hardware to simulate certain human intelligence basic theories, methods, and techniques of behavior. The authors believe that the research and development of artificial intelligence must not abandon the exploration of human beings and machines themselves. Combination and fusion can avoid the tragedy that occurs when humans learn to fly and bind the feathers of birds to their bodies. Artificial intelligence, which uses human intelligence as a reference, explores and expands problemoriented machine language systems and generates intelligence that can be deduced by human thinking $[9,10]$. This not only avoids the human-computer conflict caused by simply reengraving human thinking but also avoids certain defects that humans may have, such as personality defects and psychological defects [11].

2.1.1. Characteristics of Artificial Intelligence. By definition, the most basic feature of artificial intelligence is that it is designed by and provides services to humans. Although the name suggests, there are already some intelligent machines that specialize in manufacturing machines to serve human society. From this perspective, the characteristics of artificial intelligence have been developed from the aspects of being man-made [12]. In any case, artificial intelligence must be people-oriented.

Secondly, the realization of intelligence is based on data and learning methods. The fundamental way to realize artificial intelligence is to feed back the results through machine learning, so as to draw the processing rules of current affairs and problems $[13,14]$. This requires a certain data foundation to support artificial intelligence for machine learning. The current machine learning methods of artificial intelligence are divided into four methods: supervised learning, unsupervised learning, semisupervised learning, and reinforcement learning. They are all based on the derivation, calculation, and trial and error of a certain amount of data to improve and improve the artificial intelligence system and its stability [15].
Thirdly, they can sense the environment, can react, and can interact with people. Artificial intelligence should be able to use sensors and other devices to generate the ability to sense the external environment (including humans). Based on distance sensors, motion capture devices, voice recognizers, graphic sensors, and so forth, to obtain information about the interaction between the environment and humans, this is active; in passive information acquisition, artificial intelligence devices enter information through external input devices, such as buttons, keyboard, mouse, screen, gesture, posture, expression, force feedback, virtual reality, and augmented reality, or grab information directly from its own data warehouse [16]. Both active and passive artificial intelligence should be able to actively capture, identify, summarize and respond to environmental information, and serve humanity. Therefore, compared with humans, artificial intelligence is nonspecific, helping humans to do purely rational work that human beings are not good at or dislike. Some works may involve danger but with clear process rules, which can be completed by machines, while human beings do some more creative, insightful, imaginative, flexible, changeable, and even more perceptual work $[17,18]$.

Finally, artificial intelligence has adaptive characteristics. In short, artificial intelligence has a certain ability to adapt to the environment and can iterate and evolve itself [19]. Furthermore, artificial intelligence can analyze the information and data in the environment through machine learning and learn how to solve the problems in the task goal that it is given; that is, it has a certain degree of adaptive adjustment according to the changes in the environment, data, or tasks, and it has the ability to update the optimization model [20].

\subsection{Expert System for Mechanical Hydraulic Fault Diagnosis.} Mechanical hydraulic fault diagnosis expert system is a kind of knowledge-based application software system. It obtains professional knowledge from experts in the hydraulic field and is used to solve difficult problems that only experts in the hydraulic field can solve [21, 22]. The fault diagnosis and elimination of hydraulic systems require the application of a large number of unique expert practical experience and diagnostic strategies, the external performance of certain faults is often related to a variety of potential faults, and there are various overlaps between symptoms and causes. It often brings inconvenience to fault diagnosis [23]. Combined with engineering practice, an expert system designed for hydraulic fault diagnosis can meet the requirements of general technicians to diagnose and eliminate hydraulic system faults. It has important engineering value and is also conducive to the preservation and continuation of fault diagnosis experience and knowledge [24, 25]. The fault diagnosis of the hydraulic system is generally aimed at the specific system. By analyzing the specific failure modes and fault mechanisms of the hydraulic system, the corresponding knowledge base and reasoning mechanism are established, the extracted state detection signals are identified and classified, and the hydraulic faults are diagnosed, predicted, and monitored [26]. 
2.2.1. Characteristics of Mechanical Hydraulic Fault Diagnosis Expert System. Mechanical hydraulic fault diagnosis expert system uses the expertise provided by human experts in the field of hydraulics to simulate the thinking process of human experts and solve problems that are quite difficult for human experts [27]. Generally speaking, a high-performance system should have the following characteristics:

(1) Expert knowledge of hydraulic diagnosis: an important goal of establishing a hydraulic fault diagnosis expert system is to achieve the high-level performance of experts in solving hydraulic diagnosis tasks. The hydraulic fault diagnosis expert system can successfully solve various problems in the hydraulic field. It has the level of human experts in the field of the quality and speed of problem-solving and the ability to use heuristic rules. The fundamental reason is that expert-level knowledge is stored in the system.

(2) Expert-level solution capabilities: the construction of the inference engine of the hydraulic and fault diagnosis expert system and the selection of the control strategy must be prudent, so that the reasoning of the expert system is intelligent, reaching or exceeding the level of human experts.

(3) The ability to acquire knowledge: human experts can continuously enrich their knowledge through learning, and a high-performance hydraulic fault diagnosis expert system should also have this ability to continuously acquire knowledge.

(4) Knowledge and reasoning institutions are independent of each other: the expert system generally separates the reasoning mechanism from knowledge and makes it independent, which makes the system have good expandability and maintainability.

\subsubsection{Composition of Mechanical Hydraulic Fault Diagnosis} Expert System. The general structure of the expert system has six parts: knowledge base, inference engine, comprehensive base, database, man-machine interface, explanatory system, and knowledge acquisition subsystem (knowledge acquisition) as shown in Figure 1.

The knowledge base is where the expert system stores knowledge. The knowledge engineer interacts directly with the domain expert to collect and organize the knowledge of the domain expert, transform it into the internal representation of the system and store it in the knowledge base; the inference engine is the expert system. The core part, according to the user's problem-solving requirements and the initial data provided, uses the knowledge in the knowledge base to solve the problem and outputs the results to the user; the comprehensive database is used to store the initial data about the problem, the solution status, intermediate results, assumptions, goals, and final solution results; the knowledge acquisition subsystem acts as an interface between expert systems and domain experts and knowledge engineers when constructing and maintaining a knowledge base; and explaining the subsystem's specific problems in the execution of the entire system explain to enhance the user's understanding and trust in the system's reasoning. The human-machine interface is the medium for communication between the user and the expert system. It transforms the information that the two interact with into a form in which they can understand each other.

2.3. Implementation of the Expert System. Fault diagnosis expert system (ES) is the most widely used intelligent diagnosis system $[28,29]$. It is mainly used for complex systems without precise mathematical models or under the condition where it is difficult to establish mathematical models. The hydraulic system fault diagnosis expert system is developed based on the use of advanced sensing technology and signal processing technology. The general process of diagnosing a hydraulic system fault with an expert system is to input the fault phenomenon into a computer through a user interface. The computer then infers the cause of the fault and proposes maintenance based on the input fault phenomenon and the knowledge in the knowledge base. According to the reasoning method stored in the reasoning machine, the fault causes are inferred, and maintenance and preventive measures are proposed [30]. According to the knowledge base model and knowledge inference processing method, the implementation of the expert system consists of several modules as shown in Figure 2.

Expert systems will encounter the "bottleneck" of knowledge acquisition in the development. The knowledge base is too large and unstructured, and the solution method is single, which makes its support capacity limited. Moreover, the artificial neural network opens up new space for intelligent diagnosis of the hydraulic system. Artificial neural networks use fault tolerance, learning functions, associative memory functions, and distributed parallel information processing functions of neural networks to better solve the "bottleneck" problems of traditional methods in knowledge expression, acquisition, and parallel reasoning. In particular, it does not require a tree search, which greatly reduces the system development cycle and improves the solution efficiency. The specific application methods are applying neural network as a classifier for fault diagnosis of hydraulic system from the perspective of pattern recognition; applying neural network as a dynamic model for fault prediction of hydraulic system from the perspective of fault prediction; and applying neural network to obtain residual error from the perspective of fault detection hydraulic system.

\section{Experiments}

3.1. Experimental Data Analysis and Preprocessing. Data preprocessing is also called data-level fusion. It is a low-level fusion in information fusion systems. In this chapter, datalevel fusion is mainly the feature parameter extraction and normalization of data obtained from hydraulic systems by parallel sensors. The pump casing vibration signal and external leakage temperature signal are monitored and collected online, and then the characteristic parameters are extracted and normalized. Because the vibration signal is 


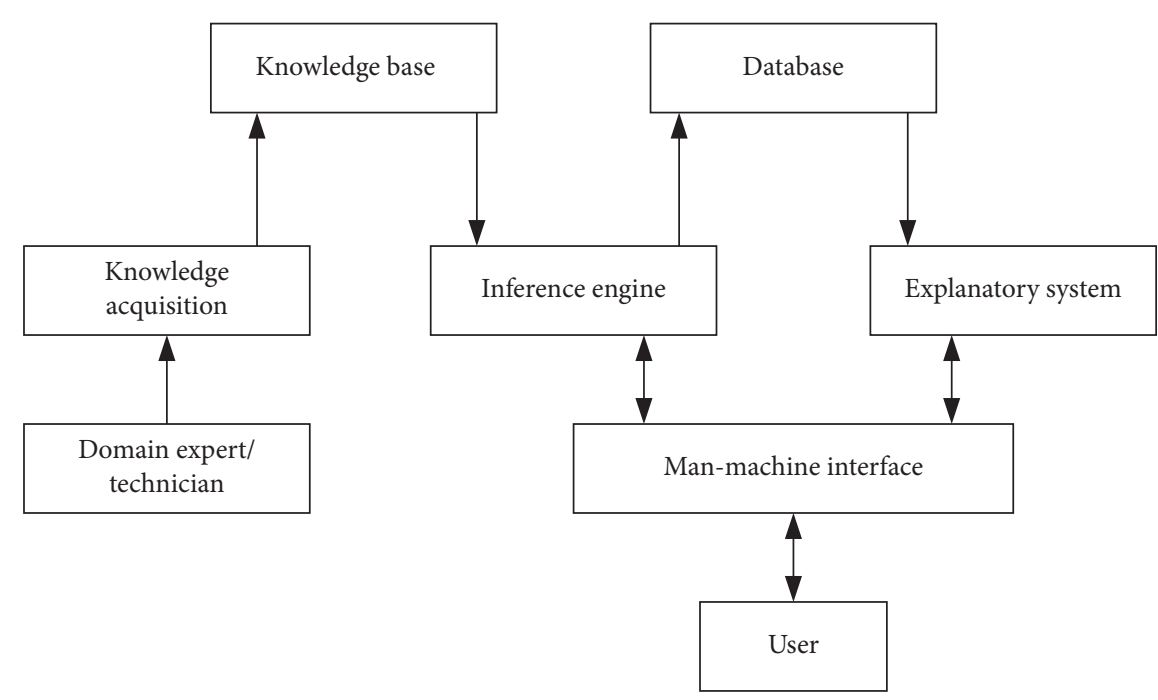

FIgURE 1: General structure of an expert system.

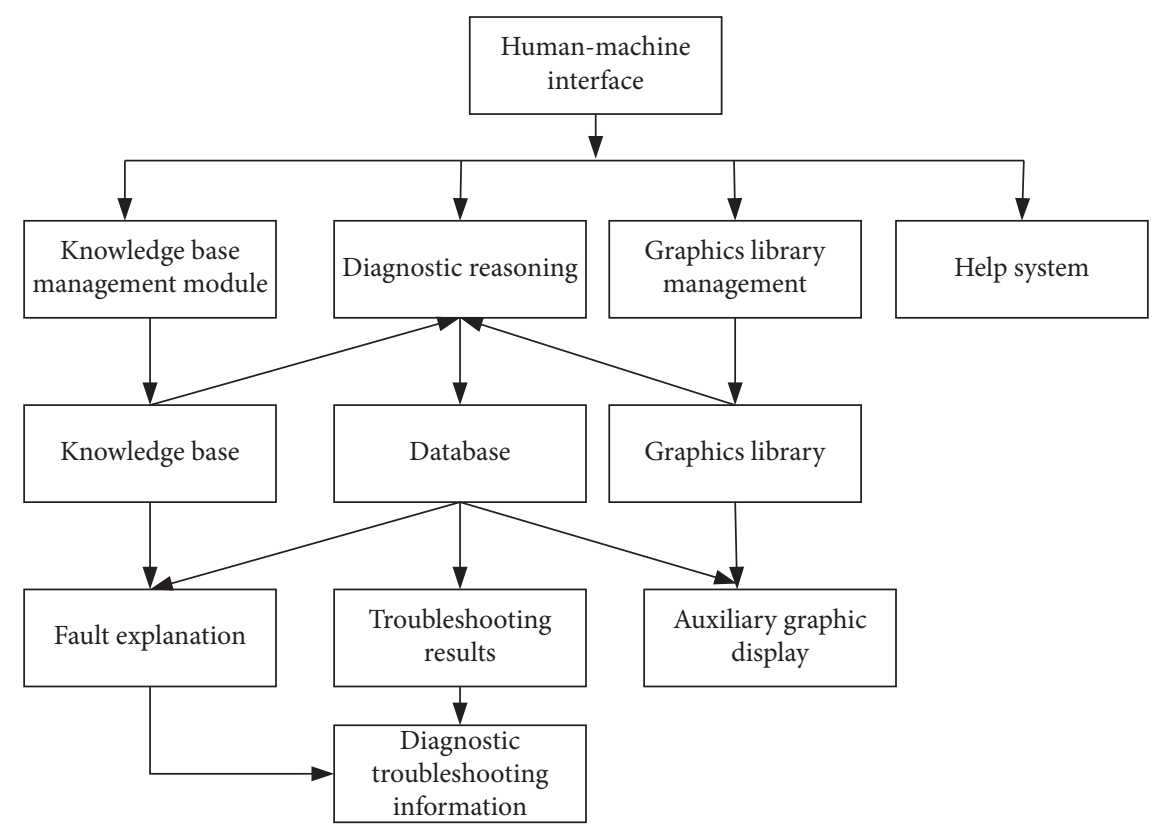

FIgURE 2: Block diagram of the hydraulic fault diagnosis expert system.

susceptible to noise pollution during the acquisition process, the collected data is converted in the amplitude range to reduce the effect of noise on the signal. Select 8 amplitude domain parameters of peak value, peak-peak value, root mean square value, peak value index, margin index, waveform index, pulse index, and kurtosis index as the characteristic parameters. Among them, the margin index, pulse index, and kurtosis index are sensitive to early faults but are unstable. The larger the kurtosis value, the more the measured system deviates from the normal state.

A set of characteristic samples of the normalized vibration signals in the three directions is shown in Table 1. Their corresponding target outputs are normal status, loose ball heads, wear of the flow plate, loose boots, cylinder wear, and bearing wear and leak seven failure modes.

\subsection{Experimental Testing with Major Instruments}

(1) Test a PC.

(2) Data acquisition card: USB-6211 plug-and-play data acquisition card from National Instruments, USA, no external power supply required, and working environment humidity of $0-45^{\circ} \mathrm{C} ; 16$ single-ended or 8-channel differential maximum sampling rate of $250 \mathrm{KS} / \mathrm{S}$ analog input, input impedance $>10 \mathrm{G} \Omega$, 
TABLE 1: Characteristic parameters of hydraulic vibration signals.

\begin{tabular}{lcccccrrr}
\hline & $X_{\max }$ & $X_{\mathrm{ff}}$ & $X_{\mathrm{ms}}$ & $S_{f}$ & $C_{f}$ & $I_{f}$ & $\mathrm{CL}_{f}$ & $K_{v}$ \\
\hline Normal & 0.6402 & 0.7153 & 0.6287 & 0.4326 & 0.6482 & 0.6447 & 0.64471 & 0.4960 \\
Loose ball head & 0.4034 & 0.3758 & 0.3674 & 0.3377 & 0.4320 & 0.4181 & 0.4193 \\
Worn flow plate & 0.3373 & 0.3886 & 0.6298 & 0.6240 & 0.3280 & 0.3096 & 0.3121 & 0.4617 \\
Loose boot failure & 0.4457 & 0.3914 & 0.6147 & 0.6140 & 0.4004 & 0.4662 & 0.4654 & 0.3633 \\
Cylinder wear & 0.5444 & 0.6166 & 0.4797 & 0.4546 & 0.3768 & 0.4488 & 0.4415 & 0.5101 \\
Cylinder wear & 0.4418 & 0.5355 & 0.3074 & 0.2877 & 0.5215 & 0.4776 & 0.4758 & 0.7458 \\
Leakage & 0.5526 & 0.4656 & 0.4630 & 0.4685 & 0.5285 & 0.5818 & 0.5867 & 0.4351 \\
\hline
\end{tabular}

common mode rejection ratio of 100 , and voltage accuracy at maximum range $<2.69 \mathrm{mV}$; and 2 counters/timers, using TTL logic level, can be used for encoder data acquisition.

(3) Power bank: RvixeRV-22000A power bank can provide $5 \mathrm{~V}$ USB output, which can also be set as 12/ $16 / 19 \mathrm{~V}$ DC output. The maximum output currents for $5 \mathrm{~V}$ USB and $12 / 16 / 19 \mathrm{~V}$ connections are $2.1 \mathrm{~A}$ and $3.5 \mathrm{~A}$, respectively, with the power capacity of $22000 \mathrm{mAh}$, which can power all sensors at the same time. The mobile power supply cooperates with a laptop computer and a USB data acquisition device. The full test system does not require an external power supply, which can prevent $220 \mathrm{~V}$ AC power from interfering with the signal.

(4) Sensors: some measurement items and selected sensors are shown in Table 2.

The dual-axis gyroscope is installed on the pallet to measure the angular velocity of the pallet arm. The sensor fixture is made of lead alloy. Compared with the mechanical part, the weight of the sensor and fixture is almost negligible, which has no effect on the swing process.

All power interfaces use a $5.5 \mathrm{~mm}$ DC power interface, and all signal lines are concentrated on the FC cable. In order to improve the common mode rejection ratio, all analog inputs use the differential signal connection. Because the notebook power supply used by the data acquisition card and the mobile power supply used by the sensor are suspended, in order to prevent the difference between the zero potentials of the two and the data acquisition card, PGA (Programming Gain Amplifier) common mode range, thus erroneous measurement may be caused by PGA saturation. Connect pull-down resistors at both ends of the signal and the GND terminal of the acquisition card. At the same time, the encoder output is HTL logic level signal, and the data acquisition card counter input is TTL level, which provides $12 \mathrm{~V}$ to $5 \mathrm{~V}$ voltage divider circuit for the encoder signal. These are all made on a simple circuit board, as the adapter board of the sensor and data acquisition card, during the experiment, only the FC cable header of the signal and the FC cable header of the data acquisition card are inserted into the corresponding horn sockets on the adapter board.

\section{Discussion}

4.1. Fault Diagnosis and Analysis. In order to verify the performance of the trained four neural networks, the historical fault data of the mechanical hydraulic system at certain three moments are processed as test samples, and the subneural networks constructed above are used for diagnosis.

First of all, take fault 2 of the flow plate wear that occurred on a certain day as an example for diagnosis. After extracting and normalizing the vibration signals in three directions when the hydraulic piston pump flow plate fails, the characteristic data obtained is shown in Table 3.

The feature vector data in Table 3 is used as a test sample, and the three subneural networks that have been trained are input for testing. The local diagnosis results are shown in Table 4.

Suppose that $\varepsilon 1=\varepsilon 2=0.5$ and $\gamma=0.1$ in the decision rule. From the diagnosis results of the three subnets in Table 4, it can be seen that, compared with the failure modes shown in Table 3, the failure can be initially judged as the wear of the flow plate, and the diagnostic output of the vibration signals in the three directions is consistent. However, it can also be found from the table that the diagnosis result in the $Y$ direction has strong ambiguity, and the reliability of fault 4 in the diagnosis result is also relatively high, reaching 0.5060 . According to the decision rules, the diagnosis result in the $Y$ direction cannot determine the status of the hydraulic system in a machinery. Therefore, in order to obtain more clear and accurate diagnosis results, it is necessary to make full use of multisensor information fusion technology to make fusion decision on different subnet diagnosis results.

The test software is written on the LabVIEW platform and includes a configuration interface and a data display interface. It collects 7 channels of signals at the same time, sets the sampling frequency of all signals to 1000, and simultaneously performs signal acquisition, electrical signal to physical signal conversion operations, data curve display, and data conversion. This is saved to TDMS file. After the test is completed, the data is sorted, and the test results show that the device operates well and the data values measured multiple times have a high degree of repeatability. The change in the angular velocity of the carrier and the system hydraulic pressure during the swing and its return is shown in Figure 3.

Most of the signal testing is done by sensors. The test signal carries useful information for research and often contains other interference noises. Due to the complexity of the mechanical hydraulic system and the influence of the surrounding noise or the misalignment of the sensor in the working process, the software and hardware in the acquisition process and other factors, the interference signal will inevitably be mixed in the test system, resulting in the fact 
TABle 2: Measurement items and sensors.

\begin{tabular}{lccc}
\hline Component & Process & Measurement item & Sensor \\
\hline Coordinator & Bouncing process & $\begin{array}{c}\text { Pendulum angular velocity } \\
\text { Hydraulic pressure }\end{array}$ & $\begin{array}{c}\text { Dual-axis gyroscope } \\
\text { Pressure sensor }\end{array}$ \\
\hline
\end{tabular}

TABLE 3: Test sample output.

\begin{tabular}{lcc}
\hline & $\begin{array}{c}\text { Worn flow plate } \\
X \text { direction }\end{array}$ & $Z$ direction \\
\hline 0.3889 & 0.3790 & 0.3803 \\
0.4676 & 0.3694 & 0.2761 \\
0.6428 & 0.3812 & 0.5512 \\
0.6359 & 0.4657 & 0.5391 \\
0.2787 & 0.3747 & 0.3982 \\
0.3341 & 0.3776 & 0.3809 \\
0.3360 & 0.3791 & 0.3828 \\
0.3655 & 0.4957 & 0.5259 \\
\hline
\end{tabular}

TABLE 4: Test sample output.

\begin{tabular}{lccc}
\hline \multicolumn{3}{c}{ Worn flow plate } & \\
Match pattern & $X$ direction & $Y$ direction & $Z$ direction \\
\hline 0 & 0.0281 & 0.0710 & 0.0808 \\
1 & 0.8422 & 0.7149 & 0.9334 \\
0 & 0.0471 & 0.0243 & 0.0444 \\
0 & 0.0105 & 0.5049 & 0.0186 \\
0 & 0.0182 & 0.0044 & 0.0283 \\
0 & 0.0304 & 0.0278 & 0.0029 \\
\hline
\end{tabular}

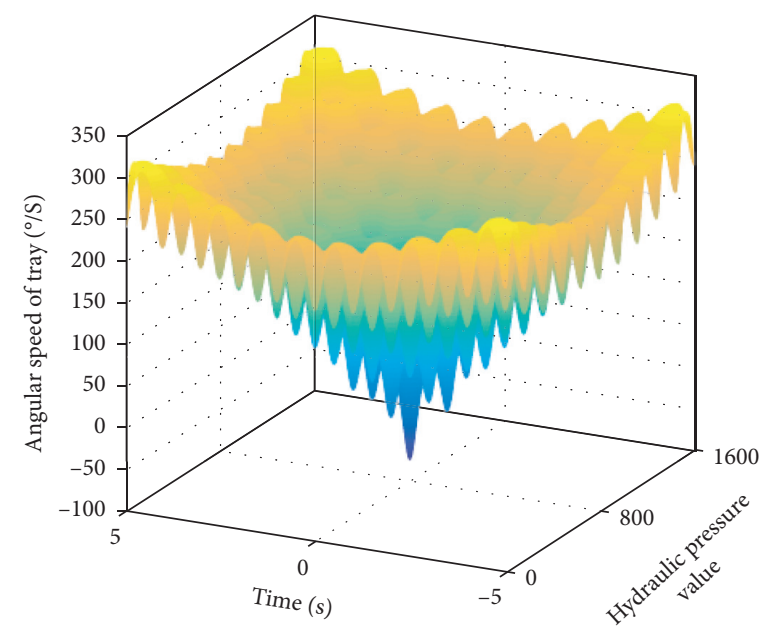

FIGURE 3: System hydraulic pressure values.

that failure cannot be reliably identified. Therefore, the first step of signal analysis and processing is to filter out the interference signal. In addition, the state signal of the actual hydraulic system is the result of the superposition of the stable signal and a large number of nonstationary signals. The rotation angular velocity of the bearing plate is a lowfrequency stable signal under normal working conditions. The vibration of the mechanical device, the connecting rod piston of the hydraulic cylinder, and so forth in the working process is random and contains nonstationary signals. Wavelet multiscale analysis, as a nonstationary signal denoising method, can accurately represent the dynamic characteristics of faults and greatly preserve the original information of the signal. Wavelet multiscale analysis is to decompose the original signal into two parts: a low-frequency approximation signal and a high-frequency detail signal. The noise signal is hidden in the high-frequency signal, and then only the low-frequency signal is continuously decomposed. Each decomposition will filter the highfrequency noise to achieve the purpose of noise reduction.

The db4 wavelet was used to denoise the angular velocity signal of the pallet during the swinging process, as shown in Figure 4.

It can be seen from the test results that the signal obtained by $\mathrm{db} 4$ wavelet noise reduction is smoother, the characteristics of different frequency bands are more obvious, there are fewer glitches, and the original signal information is basically retained. On the other hand, it can be seen that the high-frequency signal is mainly concentrated in the initial stage of the swing bomb. It is relatively stable during the subsequent action and there is not much noise interference. The reason may be that the solenoid valve was some pressure fluctuations or collisions and vibrations between components due to inertia (such as between the projectile and the tray), and whenever the speed changes (such as the beginning acceleration and end deceleration phases), the high-frequency signal is obvious, which may be the connections between components cannot be absolutely fixed and cause collision or vibration with each other.

4.2. Comparison of Experimental and Simulation Models. After the test data is obtained, combining the differences between the model and the experimental equipment, some parameters that are sensitive to the model output are selected and repeated modification attempts are made within a reasonable range to verify the correctness of the model. For example, the friction coefficient in a motion pair can be used as an influencing parameter. Friction is a common phenomenon in a motion pair. When modeling the mechanical system, the friction effects in the motion pair are often ignored, or all friction is equivalent to the transmission efficiency. Obviously, this processing method cannot accurately reflect the true situation of the system. In this paper, appropriate friction coefficients are added to different kinematic pairs, and it is found that friction has a significant effect on the system's motion and should be considered. Therefore, the friction coefficient was added when the kinematic pair was established. After referring to the relevant friction coefficient manual, the model was adjusted within a 


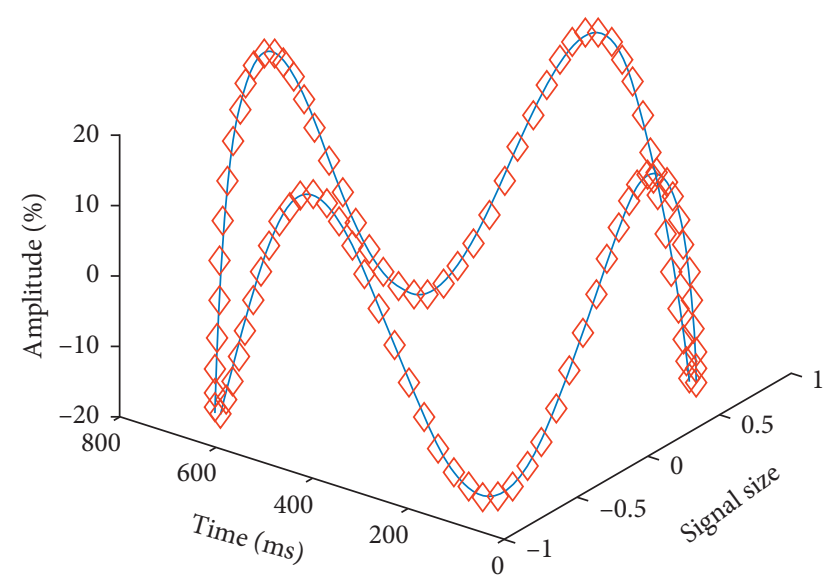

Figure 4: Wavelet denoising analysis.

reasonable range. The simulation and experiment comparison of the angular velocity of the tray is shown in Figure 5.

The dynamic simulation analysis of the mechanical model in the static state can be used to obtain the change of the angular velocity of the carrier disk at each stage. The measured curve and the simulated curve are compared. Figure 5 shows the simulation and experimental comparison of the tray angular velocity during the swing process. Due to the fixed connection between the projectile and the tray, considering the collision between the projectile and the tray, the impact oil red of the buffer section at both ends of the pendulum, and the fluctuation of the measurement signal when the impact is in place, it can be seen from the figure that the curve trend of the two is basically consistent, and the simulation results are good. Practice shows that the cosimulation model established in this case has high accuracy and can be used for fault simulation research.

4.3. Temperature Subnet Diagnosis. Usually, when the hydraulic pump fails, it is accompanied by energy loss such as loss of hydraulic energy, friction between friction pairs, and internal leakage. It can be known from the law of conservation of energy that these lost energies will be converted into other forms of energy. In the hydraulic system of pump trucks, it is mainly expressed as thermal energy. When the hydraulic pump fails, its temperature changes significantly. Measurement of the change can be used to determine the working status of the hydraulic pump.

Normalize the temperature signals of the hydraulic pump when fault 1 , fault 2 , and fault 3 occur, and enter the trained temperature subnet for testing. The diagnostic results obtained are shown in Table 5 .

It can be seen from Table 5 that the reliability of the diagnosis results of the temperature subnet is relatively high, and the fault can be diagnosed well. However, because the temperature signal is affected by the outside world, the diagnosis result of fault 1 is fuzzy, the diagnosis result of the network is inconsistent with the actual situation, and the state of the pump cannot be judged. The diagnosis results of fault 2 and fault 3 correspond to the actual fault status.

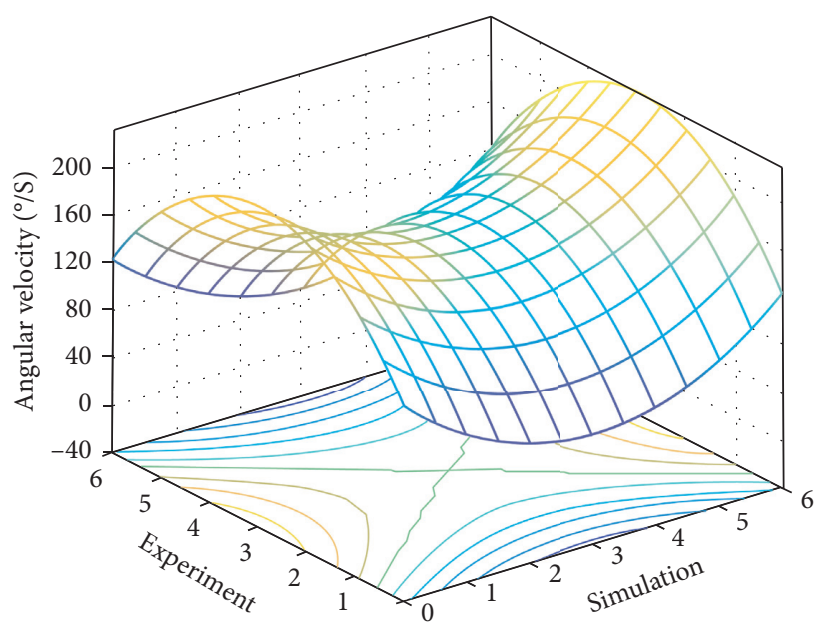

FIgURE 5: Pallet angular velocity simulation and experiment comparison.

Therefore, it is necessary to further merge the diagnosis results of the three-direction vibration subnet with the diagnosis results of the temperature subnet to obtain more accurate and high-precision diagnosis results.

After the fusion of the four lines of evidence with improved DS evidence theory, the diagnosis results are consistent with the actual fault status, the reliability is increased from $0.8533,0.7160$, and 0.9345 diagnosed by each subnet to 0.9999 , and the uncertainty is only 0.000001 . The credibility is also basically 0 . At the same time, fuzzy diagnosis results appear in the diagnosis results of the subnet in the $Y$ direction. After the subnet fusion, this kind of ambiguity is eliminated, and the accuracy and precision of diagnosis are greatly improved. For fault 1 and fault 3, the four lines of evidence are merged according to the method steps described above, and the weight coefficients of each evidence body for fault 1 and fault 3 are calculated: 0.9565, 0.8835, $0.9430,0.9005$ and $0.9428,0.9341,0.6263,0.9138$; the results obtained are shown in Figure 6.

Compared with the diagnosis results of the three subnets of the vibration signal shown in Figure 6, the diagnostic accuracy of the results of the fusion of the four lines of evidence of fault 1 and fault 3 is 0.9848 and 0.9927 , respectively, and the reliability is significantly increased, approaching $100 \%$. The uncertainty is basically 0 , which significantly improves the accuracy of fault diagnosis, eliminates misdiagnosis caused by individual sensor conflicts and inconsistent diagnosis results, and obtains a more accurate final diagnosis result.

In summary, it is known that fault diagnosis using only the fault feature information of a single sensor has low credibility, uncertainty, and sometimes even fuzzy diagnosis, inconsistency, and misdiagnosis, and the fault state cannot be correctly identified. The diagnosis method based on multisource information fusion has strong robustness and fault tolerance. After the fusion of the multisensor feature information of multiple sensors with each other, the redundant and complementary information of each sensor is fully used, the reliability and accuracy of the diagnosis are 
TABle 5: Network output for temperature subnet.

\begin{tabular}{lccccccc}
\hline & $m\left(f_{1}\right)$ & $m\left(f_{2}\right)$ & $m\left(f_{3}\right)$ & $m\left(f_{4}\right)$ & $m\left(f_{5}\right)$ & $m\left(f_{6}\right)$ & Diagnostic result \\
\hline Malfunction 1 & 0.9643 & 0.0002 & 0.0031 & 0.0000 & 1.0000 & 0.0003 & Fuzzy diagnosis \\
Malfunction 2 & 0.0105 & 0.9725 & 0.0013 & 0.0021 & 0.0012 & 0.0041 & Worn flow plate \\
Malfunction 3 & 0.0017 & 0.0018 & 0.9871 & 0.100 & 0.0120 & 0.0100 & Loose boots \\
\hline
\end{tabular}

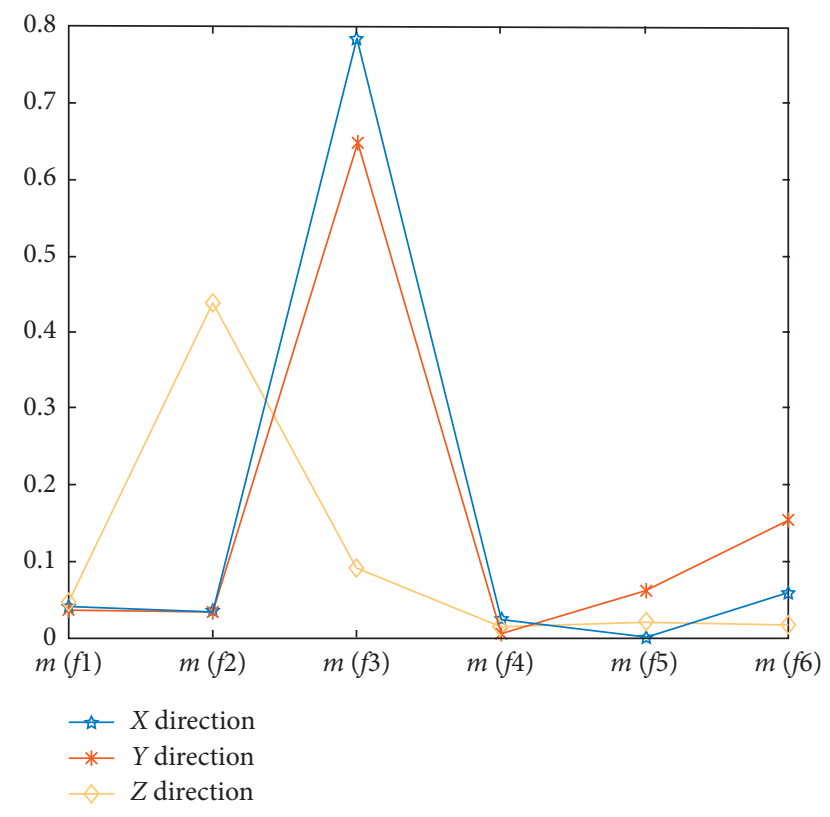

FIGURE 6: Result of each subnet fusion.

greatly improved, and the uncertainty is almost zero. At the same time, the fusion method proposed in this paper can effectively reduce the impact of conflict evidence on the final result, solve the problem of evidence conflict well, and achieve an accurate diagnosis of mechanical hydraulic system faults.

\section{Conclusions}

The rapid development of modern computer technology, detection technology, and information technology provides a technical foundation for the generation and development of intelligent fault diagnosis technology. Intelligent fault diagnosis technology has great advantages. In recent years, a lot of research has been done on the development of intelligent dynamic monitoring fault diagnosis technology at home and abroad, and it has been widely used in all walks of life. The artificial intelligence dynamic detection is introduced into the hydraulic fault diagnosis system, and the fault diagnosis model is established by using the artificial intelligence dynamic monitoring method to analyze and eliminate the fault better and faster. Research on the fault diagnosis method of the mechanical hydraulic system based on artificial intelligence dynamic monitoring and finding a better way to solve the fault diagnosis of the hydraulic system have certain practical value and application reference value for the intelligent development of construction machinery.
In this paper, the mechanical hydraulic system is introduced in detail and the failure mechanism is analyzed. Combined with the common faults of the hydraulic system and the individual faults of the mechanical hydraulic system, the FMEA table is established to fully describe the failure modes of the mechanical hydraulic system. Based on the mechanical hydraulic system as the prototype, based on the analysis of its working principle, the system was modeled using ADAMS dynamics simulation software and AMESim hydraulic simulation software. Through comparison of experimental data, it was proved that the model has good simulation results and meets the accuracy requirements. A large number of simulation calculations are performed for the three typical faults of the system. The results show that these three typical faults have different degrees of impact on the mechanical hydraulic system and can be used as fault sample data.

This paper uses functional principal component analysis and BP neural network to diagnose the above three types of fault parameters. The results show that the method can accurately identify the three listed fault modes. After diagnosing the failure mode, use the FMEA table to query the cause, effect, and use of compensation measures for the failure mode. Through the mutual cooperation of the two, it has certain practical significance for building a complete fault diagnosis system. The fault data of various fault modes in this paper are all obtained by simulation, but the high accuracy of the simulation model depends on a large number of fault statistics and test data of experimental equipment, which is lacking at the current stage. The fault modes selected in this paper are just a few typical faults that often occur in the hydraulic system of the coordinator, and they need to be improved in the future to cover a wider range of fault modes. In terms of fault diagnosis algorithms, they can be optimized or more advanced diagnosis algorithms can be selected to meet the growing demand.

\section{Data Availability}

No data were used to support this study.

\section{Conflicts of Interest}

The authors declare that there are no conflicts of interest regarding this paper.

\section{Authors' Contributions}

All authors have read the manuscript and approved before submitting it to the journal. 


\section{References}

[1] G. Wang, X. Zhang, and S. Zhu, "Hydraulic failure diagnosis of tractor hydro-mechanical continuously variable transmission in shifting process," Transactions of the Chinese Society of Agricultural Engineering, vol. 31, no. 6, pp. 25-34, 2015.

[2] V. A. Dergachev, A. N. Gorban, A. A. Rossiev et al., "The filling of gaps in geophysical time series by artificial neural networks," Radiocarbon, vol. 43, no. 2, pp. 365-371, 2016.

[3] C. Lu, Y. Wang, M. Ragulskis, and Y. Cheng, "fault diagnosis for rotating machinery: a method based on image processing," PLos One, vol. 11, no. 10, Article ID e0164111, 2016.

[4] A. Lino, Á. Rocha, and A. Sizo, "Virtual teaching and learning environments: automatic evaluation with artificial neural networks," Cluster Computing, vol. 22, no. 4, pp. 1-11, 2019.

[5] M. Andreatta and M. Nielsen, "Gapped sequence alignment using artificial neural networks: application to the MHC class I system," Bioinformatics, vol. 32, no. 4, pp. 511-517, 2015.

[6] B. Tarawneh, "Predicting standard penetration test $\mathrm{N}$-value from cone penetration test data using artificial neural networks," Geoscience Frontiers, vol. 8, no. 1, pp. 199-204, 2017.

[7] S. Marco Siniscalchi and V. Mario Salerno, "Adaptation to new microphones using artificial neural networks with trainable activation functions," IEEE Transactions on Neural Networks \& Learning Systems, vol. 28, no. 8, pp. 1-7, 2016.

[8] X. Lu and J. Fei, "Velocity tracking control of wheeled mobile robots by iterative learning control," International Journal of Advanced Robotic Systems, vol. 13, no. 3, p. 1, 2016.

[9] S. Lemeš, D. Štrbac, and C. Malik, "Using industrial robots to manipulate the measured object in CMM," International Journal of Advanced Robotic Systems, vol. 10, no. 281, pp. 1-9, 2013.

[10] J. J. Ye, "Artificial intelligence for pathologists is not near- it is here: description of a prototype that can transform how we practice pathology tomorrow," Archives of Pathology \& Laboratory Medicine, vol. 139, no. 7, pp. 929-935, 2015.

[11] L. D. Raedt, K. Kersting, S. Natarajan, and D. Poole, "Statistical relational artificial intelligence: logic, probability, and computation," Synthesis Lectures on Artificial Intelligence and Machine Learning, vol. 10, no. 2, pp. 1-189, 2016.

[12] M. Hutson, "Artificial intelligence faces reproducibility crisis,” Science, vol. 359, no. 6377, pp. 725-726, 2018.

[13] H. Citakoglu, "Comparison of artificial intelligence techniques via empirical equations for prediction of solar radiation," Computers and Electronics in Agriculture, vol. 118, pp. 28-37, 2015.

[14] S. Price and P. A. Flach, "Computational support for academic peer review," Communications of the Acm, vol. 60, no. 3, pp. 70-79, 2017.

[15] C. Cath, S. Wachter, and B. Mittelstadt, "Artificial intelligence and the 'good society': the US, EU, and UK approach," Science \& Engineering Ethics, vol. 24, no. 7625, pp. 1-24, 2017.

[16] L. Roger, "Software engineering, artificial intelligence, networking and parallel/distributed computing," Local Government Studies, vol. 209, no. 5, 2016.

[17] H. Ashrafian, A. Darzi, and T. Athanasiou, "A novel modification of the Turing test for artificial intelligence and robotics in healthcare," The International Journal of Medical Robotics and Computer Assisted Surgery, vol. 11, no. 1, pp. 38-43, 2015.

[18] H. Jaeger, "Deep neural reasoning," Nature, vol. 538, no. 7626, pp. $467-468,2016$.
[19] S. Singh, A. Okun, and A. Jackson, "Learning to play Go from scratch," Nature, vol. 550, no. 7676, pp. 336-337, 2017.

[20] H. Ashrafian, "AIonAI: a humanitarian law of artificial intelligence and robotics," Science and Engineering Ethics, vol. 21, no. 1, pp. 29-40, 2015.

[21] S. Yeung, N. L. Downing, L. Fei-Fei, and A. Milstein, "Bedside computer vision - moving artificial intelligence from driver assistance to patient safety," New England Journal of Medicine, vol. 378, no. 14, pp. 1271-1273, 2018.

[22] M. Kibria and G. Porto, "Big data analytics, machine learning and artificial intelligence in next-generation wireless networks," IEEE Access, vol. 99, 2017.

[23] J. Young and H. Sun Park, "A computer-aided diagnosis system using artificial intelligence for the diagnosis and characterization of thyroid nodules on ultrasound: initial clinical assessment," Thyroid, vol. 27, no. 4, p. 546, 2017.

[24] J. Qiu, "Research and development of artificial intelligence in China," National Science Review, vol. 3, no. 4, pp. 538-541, 2016.

[25] A. Jalalkamali, M. Moradi, and N. Moradi, "Application of several artificial intelligence models and ARIMAX model for forecasting drought using the Standardized Precipitation Index," International Journal of Environmental Science and Technology, vol. 12, no. 4, pp. 1201-1210, 2015.

[26] P. Sernani, A. Claudi, and A. F. Dragoni, "Combining artificial intelligence and NetMedicine for ambient assisted living," International Journal of E-Health and Medical Communications, vol. 6, no. 4, pp. 62-76, 2015.

[27] B. B. Nair and V. P. Mohandas, "Artificial intelligence applications in financial forecasting-a survey and some empirical results," Intelligent Decision Technologies, vol. 9, no. 2, pp. 99-140, 2015.

[28] S. Das, A. Dey, A. Pal, and N. Roy, "Applications of artificial intelligence in machine learning: review and prospect," International Journal of Computer Applications, vol. 115, no. 9, pp. 31-41, 2015.

[29] N. Dudhwala, K. Jadhav, and P. Gabda, "Prediction of stock market using data mining and artificial intelligence," International Journal of Computer Applications, vol. 134, no. 12, pp. 9-11, 2016.

[30] F. Yu, N. Cui, and Q. Zhang, "Comparison of artificial intelligence and empirical models for estimation of daily diffuse solar radiation in North China Plain," International Journal of Hydrogen Energy, vol. 42, no. 21, pp. 14418-14428, 2017. 